\title{
Diacronie
}

Studi di Storia Contemporanea

$N^{\circ} 9,1 \mid 2012$

Quando la classe operaia andava in paradiso

\section{Nazionalità e lotta di classe}

La Nuova Sinistra e i nazionalismi periferici

\section{Paolo Perri}

\section{(2) OpenEdition}

Journals

\section{Edizione digitale}

URL: http://journals.openedition.org/diacronie/3039

DOI: 10.4000/diacronie.3039

ISSN: 2038-0925

Editore

Association culturelle Diacronie

Notizia bibliografica digitale

Paolo Perri, « Nazionalità e lotta di classe », Diacronie [Online], № 9, 1 | 2012, documento 11, Messo online il 29 janvier 2012, consultato il 25 avril 2019. URL : http://journals.openedition.org/

diacronie/3039; DOI : 10.4000/diacronie.3039 


\title{
Diacronie
}

\section{1/}

\section{Nazionalità e lotta di classe.}

\section{La Nuova Sinistra e i nazionalismi periferici}

\author{
Paolo PERRI*
}

L'articolo si propone di affrontare le relazioni intercorse tra il marxismo italiano $e i$ nazionalismi periferici, attraverso la rilettura di una storia politica che affonda le sue radici nel Congresso di Colonia del Partito Comunista d'Italia e prosegue in sordina fino allo scioglimento di Democrazia Proletaria. Il Programma d'Azione approvato a Colonia nel 1931, che prevedeva la realizzazione della Federazione delle Repubbliche Socialiste e Soviettiste d'Italia, sancì l'inizio di una tormentata e ambigua relazione tra il marxismo nostrano e il diritto all'autodeterminazione dei popoli. Subito accantonate dal movimento comunista ufficiale, queste tematiche riemergeranno periodicamente nei dibattiti della sinistra rivoluzionaria. Dai rapporti di Lotta Continua e Potere operaio con gli indipendentisti baschi e irlandesi, fino al riconoscimento ufficiale delle nazionalità periferiche italiane da parte di Democrazia Proletaria, sono molti gli elementi che invitano ad un'accurata riflessione su questi temi. L'analisi delle scelte e delle lotte condotte in nome del diritto all'autodeterminazione dalla Nuova Sinistra italiana potrebbe consentire una maggiore comprensione di un rapporto, quello tra socialismo e nazionalismo, talmente complesso da essere stato troppo spesso travisato. 


$$
\begin{aligned}
& 1 \text { nazionalismo è stato una scomoda anomalia per la teoria } \\
& \text { marxista e, proprio per tale motivo, è stato escluso più che } \\
& \text { affrontato }{ }^{1} \text {, scriveva Benedict Anderson nelle prime pagine del } \\
& \text { suo notissimo testo sull'origine di un fenomeno, quello }
\end{aligned}
$$

nazionalista appunto, capace di coniugarsi nel corso della storia con le più diverse teorie politiche. $\mathrm{Al}$ "fascino nazionalista" non resistette neppure il movimento operaio internazionale, e il rischio che la storiografia marxista evitasse volontariamente di riprendere la discussione sulla questione, spinse il celebre storico statunitense ad ammonire la comunità scientifica sui rischi insiti in una scelta tanto arbitraria quanto dannosa. La rimozione pressoché totale di una pagina, seppur complessa e sotto certi aspetti ambigua, della storia del marxismo avrebbe causato, infatti, un grave danno alla storia del movimento operaio dell'Europa contemporanea e non solo. L'origine di questo rapporto, spesso troppo frettolosamente valutato come scomodo o marginale, affonda le radici nei grandi dibattiti che portarono al collasso della Seconda Internazionale Socialista ${ }^{2}$. I grandi argomenti al centro delle discussioni in seno al più importante organismo del socialismo mondiale non interessarono direttamente l'Italia: il Partito Socialista Italiano, difatti, condannò immediatamente il compromesso con le diverse borghesie nazionali cui giunsero i socialisti tedeschi, austriaci e francesi. Il marxismo italiano, però, aveva soltanto rimandato l'appuntamento con il nuovo ingombrante protagonista della storia mondiale, $\mathrm{e}$ di lì a poco il tema dell'autodeterminazione dei popoli e delle nazioni tornerà a suscitare un certo interesse, sebbene in tono minore rispetto a paesi quali la Spagna o la Yugoslavia.

\footnotetext{
${ }^{1}$ ANDERSON, Benedict, Comunità immaginate: Origini e fortuna dei nazionalismi, Roma, Manifestolibri, 2004, p. 25.

${ }^{2}$ Si vedano a riguardo: COLE, George D. H., Storia del pensiero socialista, Bari, Laterza, 1968; DOLLÉANS, Edouard, Storia del movimento operaio 1820-1951, Firenze, Sansoni, 1968; HAUPT, George, Il fallimento della Seconda Internazionale, Roma, Savelli, 1970; HAUPT, George, La Seconda Internazionale, Firenze, La Nuova Italia, 1973; SALSANO, Alfredo (a cura di), La Seconda Internazionale, Roma-Bari, Laterza, 1981.
} 


\section{Il Congresso di Colonia e il dibattito nel Partito Comunista}

L'ingresso ufficiale della querelle nazionalista, o nazionalitaria ${ }^{3}$, nel dibattito marxista del nostro paese potrebbe essere ricondotto al congresso clandestino che il Partito Comunista d'Italia tenne nel 1931 in Germania, presso Colonia. L'influenza degli interrogativi posti da Antonio Gramsci sull'eredità delle funzioni politico-culturali degli stati pre-unitari all'interno del nuovo stato italiano non potevano non trovare eco nelle accese discussioni in atto nel partito comunista4. Nell'appello per l'approvazione del Programma d'azione del partito, infatti, si rivendicò, per la prima volta, «il diritto all'autodecisione delle minoranze tedesche, slovene e croate e l'indipendenza dei popoli delle colonie italiane (Libia ed Eritrea); e l'autogoverno autonomistico per le popolazioni del Mezzogiorno e delle isole»5. Un enunciato di questo tipo rappresentò un elemento di estremo rilievo da un punto di vista programmatico, ponendo un forte accento sulla possibilità di realizzare un futuro stato socialista, autonomistico e federativo. Sulla scorta dell'interpretazione degli scritti gramsciani, e del dibattito alimentato dalle posizioni assunte da Luigi Longo, che nel 1927 si spinse fino a paventare la necessità della secessione del meridione ${ }^{6}$, si giunse alla definitiva approvazione del Programma d'azione:

Allo scopo di accelerare lo sviluppo economico, politico e culturale del Mezzogiorno, della Sicilia e della Sardegna e di soddisfare le aspirazioni delle masse lavoratrici, la rivoluzione proletaria promuoverà una particolare

\footnotetext{
3 Il termine "nazionalitario", mutuato dagli studi di Arnold Van Gennep, indica le rivendicazioni di quei territori che si considerano coloniali e che lottano per una qualche forma di indipendenza. Cfr. VAN GENNEP, Arnold, Traité comparatif des nationalités, Paris, CthsComité des Travaux, 1995. Sulle diverse interpretazioni del termine si vedano inoltre: TRONCONI, Filippo, I partiti etnoregionalisti: La politica dell'identità territoriale in Europa occidentale, Bologna, Il Mulino, 2009; MELLUCCI, Alberto, DIANI, Mario, Nazioni senza stato: I movimenti etnico-nazionali in Occidente, Milano, Feltrinelli, 1992; SALVI, Sergio, Patria e Matria, Firenze, Vallecchi, 1978.

4 GRAMSCI, Antonio, Sul Risorgimento, Roma, Editori Riuniti, 1980; ID., La questione meridionale, Roma, Editori Riuniti, 1972.

5 SPRIANO, Paolo, Storia del Partito comunista italiano, vol. II, Torino, Einaudi, 1975, p. 314.

${ }^{6}$ I dibattiti che animarono il partito nel 1927, che Spriano definirà "l'anno terribile", videro Luigi Longo impegnato in un'aspra polemica sul corso che la lotta rivoluzionaria avrebbe dovuto prendere in Italia: "C'è un motivo gramsciano importante anche nella polemica di Longo: la rivendicazione di una piattaforma federativa, autonomistica, specie per il Sud, quasi separatistica. Longo, proprio richiamandosi alle tesi del III congresso sulla questione nazionale e coloniale, sostiene che la carta federativa è una grande carta per la rivoluzione proletaria italiana. [...] Si ha, del resto - egli aggiunge - tutto l'interesse a che le forze e le tendenze separatiste si sviluppino. Col loro sviluppo esse indeboliscono l'unità dello Stato borghese». Cfr. SPRIANO, Paolo, op. cit., vol. II, p. 107.
} 
organizzazione autonoma politico-amministrativa di queste regioni, sino alla costituzione di repubbliche socialiste e soviettiste del Mezzogiorno d'Italia, della Sicilia e della Sardegna, nella Federazione delle Repubbliche Socialiste e Soviettiste d'Italia. La rivoluzione proletaria darà alle minoranze nazionali il diritto di disporre di sé stesse sino alla separazione, realizzerà questo diritto nel modo più assoluto, $\mathrm{e}$ libererà tutte le popolazioni coloniali dall'oppressione dell’imperialismo italiano.7

Si combinò, così, una particolare interpretazione della questione meridionale all'ispirazione di tipo federale proveniente dal modello sovietico.

Nel testo si fa espresso riferimento alle colonie italiane d'oltremare, lasciando avvolta, però, da una certa vaghezza la questione delle nazionalità periferiche presenti all'interno dello stesso stato italiano. L'identificazione delle cosiddette questioni nazionali periferiche ha rappresentato, e sotto certi aspetti rappresenta ancora oggi, un tema sul quale molto è stato scritto. Affrontare una questione del genere comporta implicitamente l'utilizzo e l'analisi di una terminologia sovente foriera di polemiche e di interpretazioni partigiane e fuorvianti. Evitando di addentrarmi nell'interminabile dibattito sull'utilizzo di concetti come etnia, etnonazionalismo o etnoregionalismo, mi limiterò ad annoverare tra le probabili nazionalità periferiche presenti all’interno dei confini italiani, la Sardegna ed il Friuli ${ }^{8}$. In base alla comparazione e all'analisi di fattori culturali, linguistici e storico-politici, infatti, entrambi i contesti indicati presentavano le caratteristiche adatte alla nascita pressoché immediata di movimenti di ispirazione nazionalitaria, di tipo autonomista o indipendentista ${ }^{9}$. Con il Congresso di Colonia, quindi, la discussione sulla presunta alterità di alcune aree all’interno dei confini patri, fece il proprio ingresso ufficiale nella storia del marxismo del nostro paese, inducendo anche il movimento comunista ad una approfondita riflessione a riguardo. La "vacanza

\footnotetext{
7 Testo completo in Programma d'azione del PCI. Cfr. Il IV congresso del Partito comunista d'Italia (aprile 1931), Tesi e risoluzioni, Parigi, Edizioni di cultura sociale, 1931. Si veda anche SPRIANO, Paolo, op. cit., vol. II, pp. 315-316; VIDOTTO, Vittorio, Il Partito Comunista Italiano dalle origini al 1946, Bologna, Cappelli, 1974, pp. 130-138.

8 Per il dibattito sulla presunta origine etnica delle nazioni si rimanda a HOBSBAWM, Eric J., Nazioni e nazionalismi dal 1780, Torino, Einaudi, 1991; SMITH, Anthony D., Nazioni $e$ nazionalismo nell'era globale, Trieste, Asterios, 2000; GRILLI DI CORTONA, Pietro, Stati, nazioni e nazionalismi in Europa, Bologna, Il Mulino, 2003; KELLAS, James G., Nazionalismi ed etnie, Bologna, Il Mulino, 1993; MINAHAN, James, Nations Without States: A Historical Dictionary of Contemporary National Movements, Westport, Greenwood Press, 1996; ROKKAN, Stein, URWIN, Derek, Center Periphery Structures in Europe, Frankfurt-New York, Campus Verlag, 1987; RUSCONI, Gian Enrico, Se cessiamo di essere una nazione: Tra etnodemocrazie regionali e cittadinanza europea, Bologna, Il Mulino, 1993; SALVI, Sergio, Le nazioni proibite, Firenze, Vallecchi, 1973.

9 Sul dibattito a riguardo: RUSCONI, Gian Enrico, op. cit.; MELLUCCI, Alberto, DIANI, Mario, op. cit.; GRILLI DI CORTONA, Pietro, op. cit.; CACIAGLI, Massimo, Regioni d'Europa: Devoluzioni, regionalismi, integrazione europea, Bologna, Il Mulino, 2003; NEVOLA, Gaspare, Altre Italie: Identità nazionali e regioni a statuto speciale, Roma, Carocci, 2003.
} 
nazionalitaria" del partito comunista, come la definì il linguista Sergio Salvi ${ }^{10}$, si interromperà presto, e la conversione patriottica in atto all'interno del Comintern, che proprio negli anni '40 giunse all'apice, influenzò profondamente anche il partito italiano. Al termine del secondo conflitto mondiale questo radicale cambio di rotta risultò evidente a tutti i livelli e il "partito nuovo" - che intanto aveva modificato il proprio nome in Partito Comunista Italiano - accantonò definitivamente le posizioni assunte in precedenza sul futuro assetto dello stato ${ }^{11}$. A sancire definitivamente il nuovo corso ci pensò il programma per la Costituente, approvato nel 1947 dal V Congresso: «Preoccupato di difendere e rinsaldare l'unità politica e morale della nazione, il Partito comunista è contrario ad ogni forma di organizzazione federativa dello stato, poiché vede in essa un pericolo per l'unità così difficilmente e tardi conquistata» ${ }^{12}$.

Della Federazione delle Repubbliche Socialiste e Soviettiste d'Italia si era perso anche solo il ricordo. Se si tiene conto della tardiva proposta di ampia autonomia formulata per Sardegna e Sicilia soltanto alcuni anni dopo, il quadro scelto dai comunisti italiani risultò essere, senza possibilità di dubbio, quello dello stato unitario e centralista. Una scelta, questa, che comportò l'immediato isolamento di quanti chiedevano di riportare al centro della discussione del partito le questioni di carattere nazionalitario presenti all'interno del paese.

Come accennato in precedenza, l'individuazione immediata delle nazionalità periferiche in Italia non è cosa facile. Lo stato italiano riconosce lo status di minoranze ad alcune varianti linguistiche presenti sul territorio nazionale come i valdostani di lingua francese, i sudtirolesi di lingua tedesca, gli sloveni della Venezia Giulia e le sacche linguistiche ladine presenti sull'arco alpino, alle quali si aggiungono le comunità albanesi nel meridione, quelle greche in Salento e Calabria, le croate in Molise, le occitane in Calabria e quelle catalane in Sardegna. Trattandosi, però, di comunità generalmente molto piccole e lontane dal territorio della nazionalità-madre, anche se è facile designarle come comunità linguistico-culturali, riesce un po' troppo complesso farle rientrare nella categoria delle nazionalità periferiche. Un discorso differente meritano invece i movimenti nati nei due contesti periferici precedentemente indicati.

Tra la fine degli anni '40 e l'inizio dei '50 si assistette in Friuli e in Sardegna alla ricomparsa di istanze autonomistiche, che in alcuni casi diedero vita a marginali fenomeni separatisti. Nel 1947 nacque il primo movimento autonomista friulano che, seppur confinato fondamentalmente nell'ambito della tutela del patrimonio linguistico-

${ }^{10}$ SALVI, Sergio, Patria e Matria, Firenze, Vallecchi, 1978, p. 126.

${ }^{11}$ SPRIANO, Paolo, op. cit., vol. V, pp. 497-498.

12 VIDOTTO, Vittorio, op. cit., pp. 410-411. 
culturale, diede vita a vivaci polemiche con il Partito Comunista Italiano, partito del quale alcuni degli animatori dell'autonomismo erano o erano stati membri ${ }^{13}$. A condurre parte di questa polemica fu, infatti, un giovane intellettuale marxista, Pier Paolo Pasolini. In un articolo apparso su «Libertà», un periodico autonomista, nel gennaio del 1947, proprio Pasolini criticò fermamente l'atteggiamento del PCI sull'autonomia regionale friulana:

\begin{abstract}
In conclusione, ci dispiace di assistere all'opposizione della Federazione comunista udinese alla nostra autonomia, non solo perché sostiene la propria tesi piuttosto superficialmente, ma perché, andando verso una sicura sconfitta e pronunciandosi contro i sentimenti friulani della maggior parte degli iscritti al partito, non fa altro che procurarsi dell'impopolarità: lo dimostra il fatto che nelle votazioni comunali succede assai spesso che i consiglieri comunisti si astengono, dimostrando con questo, senza venir meno alla loro disciplina, di essere inclini al pieno riconoscimento del Friuli. ${ }^{14}$
\end{abstract}

Nonostante le pressioni da parte di giovani dirigenti e militanti locali, le orecchie del Partito Comunista rimasero ostinatamente sorde alle rivendicazioni provenienti dal Friuli, e l'atteggiamento non mutò con il trascorrere degli anni. Un discorso analogo può essere fatto per la Sardegna. Benché sull'isola il movimento autonomista fosse molto più antico e radicato di quello friulano, le istanze apertamente separatiste non trovarono mai un terreno particolarmente fertile. Negli anni del secondo conflitto mondiale la forza sardista, tra quelle di ispirazione marxista, più rilevante fu il Partito Comunista di Sardegna (PCS) ${ }^{15}$. Nato subito dopo l'inizio del conflitto, su iniziativa di Antonio Cassitta ${ }^{16}$, il movimento basava il proprio programma sulla constatazione che «la Sardegna ha proprie caratteristiche inconfondibili con le altre regioni d'Italia» ${ }^{17} \mathrm{e}$ propugnava «la costruzione di una repubblica socialista e federativa in cui la Sardegna

\footnotetext{
${ }^{13}$ Sull'autonomismo friulano si vedano: NEVOLA, Gaspare, op. cit.; ELLERO, Gianfranco, DAF: Dizionario Autonomistico Friulano, Codroipo, Istitût Ladin-Furlan Pre Checo Placerean, 2007; SALVI, Sergio, Le nazioni proibite, cit.; PASCHINI, Pio, Storia del Friuli, Udine, Arti Grafiche Friulane, 2003; STRASSOLDO, Raimondo, Lingua, identità, autonomia. Ricerche e riflessioni sociologiche sulla questione friulana, Campoformido, Ribis, 1996.

14 PASOLINI, Pier Paolo, «Sulle aspirazioni friulane», Libertà, 26 Gennaio 1947 in PASOLINI, Pier Paolo, Saggi sulla politica e sulla società, Milano, Mondadori, 2005.

${ }^{15}$ La decisione di non approfondire la complessa storia politica del Partito Sardo d'Azione è dettata esclusivamente dal carattere dell'articolo.

16 Militante marxista ed ex ufficiale della Brigata Sassari, condannato dal tribunale militare italiano per avere fraternizzato pubblicamente con i soldati austriaci in pieno conflitto durante la Prima guerra mondiale.

${ }_{17}$ SALVI, Sergio, op. cit., p. 577.
} 
sarà inserita come repubblica autonoma sarda»18. Nel 1945 il PCI riuscì ad assorbire il PCS e l'ipotesi nazionalitaria cadde definitivamente. La discussione su questi temi rimase confinata ai dibattiti interni delle federazioni comuniste locali fino all'esplodere degli anni della contestazione ${ }^{19}$.

\section{2. "Guerriglia nel cuore dell'Europa". La sinistra rivoluzionaria e il diritto all'autodeterminazione}

Nel periodo che va dal 1968 in poi, infatti, i temi legati alle nazionalità periferiche, o proibite come si diceva allora, dell'Europa occidentale ritornarono d'attualità. La nuova sinistra, o sinistra rivoluzionaria, si riapproprierà delle questioni nazionali irrisolte interpretandole alla luce del pensiero neo-marxista e della nuova realtà popolare e di classe. La comparsa di movimenti che, pur dichiarandosi rivoluzionari, lottavano per il conseguimento dell'indipendenza nazionale in diverse aree d'Europa, destò subito un particolare interesse tra i "gruppi". Il revival in atto, percepito quasi esclusivamente come un fenomeno estraneo alle dinamiche politiche italiane, portò Lotta Continua e Potere Operaio a dedicare una crescente attenzione a quanto, proprio all'inizio degli anni '70, stava accadendo in Irlanda del Nord e nei Paesi Baschi, non trascurando, tra l'altro, la contemporanea riorganizzazione del nazionalismo catalano, bretone, corso e occitano. Sotto silenzio restavano invece le presunte questioni irrisolte sul suolo patrio. La militarizzazione di alcune frange dei movimenti indipendentisti presenti in diversi stati europei, e il coevo spostamento a sinistra di alcuni di essi ${ }^{20}$, non potevano non

\footnotetext{
${ }_{18}$ Ibidem.

19 Successivamente la questione sarda tornerà ad agitare il marxismo italiano. La prima forma embrionale eversiva in Sardegna può farsi risalire alla seconda metà degli anni '6o, all'epoca del progetto di Gian Giacomo Feltrinelli mirante a trasformare l'isola in una "Cuba del Mediterraneo", insediando nella regione un gruppo guerrigliero legato ai Gruppi di Azione Partigiana, sorta di embrionale avanguardia militare di stampo marxista-leninista, che avrebbe dovuto indirizzare le istanze autonomiste locali verso una strategia di ribellione rivoluzionaria, con la collaborazione anche di criminali comuni come Graziano Mesina. Cfr. SOTGIU, Girolamo, La Sardegna negli anni della Repubblica, Roma-Bari, Laterza, 1996.

${ }^{20} \mathrm{Nel}$ corso degli anni si tingeranno di varie tonalità di rosso diversi movimenti indipendentisti, dall'Irlanda fino alla Bretagna: l'Irish Republican Army (IRA), che nel dicembre del 1969 si divise in Official e Provisional, si proclamò sempre una forza socialista, e la scissione fu in parte frutto di una profonda divergenza proprio sul tipo di lotta da portare avanti; Euskadi Ta Askatasuna (ETA) spinse ancora più avanti la propria adesione al marxismo, replicando in tutto e per tutto le dinamiche all'origine delle spaccature tra i diversi gruppi della sinistra rivoluzionaria europea (la divisione del 1973 tra ETA-Milis ed ETA-PoliMilis fu condita da un continuo scambio d'accuse di avventurismo, trotskismo, maoismo ecc.); il Front de Libération de la Bretagne (FLB) bretone, che nel 1972 diventerà a seguito di una scissione l'Armée Républicaine Bretonne (ARB), e il Free Wales Army gallese. A riguardo si vedano: ENGLISH, Richard, La vera storia dell'IRA: Il nazionalismo, la violenza, il socialismo, la religione e $i$ segreti della lotta tra Irlanda e Gran Bretagna, Roma, Newton \& Company, 2004; Ó BROIN,
} 
esercitare un certo fascino sui giovani militanti dei gruppi della sinistra rivoluzionaria ${ }^{21}$. Alcune parole d'ordine lanciate dall'IRA in quegli anni, come "prendiamoci le città", richiamavano implicitamente gli slogan cari a un movimento come Lotta Continua (LC), e il modello di lotta metropolitana portato avanti nel nord dell'Irlanda risultava indubbiamente più applicabile al contesto italiano rispetto a quello vietnamita $o$ mediorientale. Da un'analisi della pubblicistica del gruppo si evince un meticoloso impegno, da parte dei responsabili della commissione internazionale, nel fornire accuratissimi resoconti sulla situazione politica in Ulster e in altre zone d'Europa attraversate da simili fermenti. La definizione del conflitto irlandese non più nei termini di uno scontro meramente religioso, unita all'approfondimento puntuale delle istanze portate avanti dalle diverse parti in causa, forniranno un importante contributo alla riflessione storica e politica di quei giovani militanti e non solo ${ }^{22}$. Dallo spoglio degli articoli comparsi su «Lotta Continua» dall'inizio del 1971, emerge con chiarezza la vicinanza dei corrispondenti italiani ai vertici del movimento repubblicano. Una prima conferma potrebbe essere rappresentata dalla notizia di un incontro segreto, tenutosi nella contea di Armagh ${ }^{23}$, tra membri dell'IRA Provisional, dell' ETA e dell'Armée Républicaine Bretonne (ARB). In un articolo pubblicato nella primavera del 1972, infatti, si specificava come la finalità dell'incontro fosse stata la conclusione di un accordo generale di stampo politico-militare, atto a coordinare la lotta armata di massa in Europa ${ }^{24}$. La notizia di questo accordo, effettivamente stretto in quella occasione, non diverrà di dominio pubblico in Gran Bretagna prima di qualche mese ${ }^{25}$ e verrà confermata dai diretti interessati con un comunicato congiunto soltanto due mesi dopo ${ }^{26}$. La serie di incontri e conferenze organizzati in Italia in quello stesso aprile del 1972, cui prese parte Dermot Kelly, presentato come rappresentante del Sinn Féin ma in realtà membro attivo dell'IRA Provisional, rappresenterebbe una ulteriore conferma dello stretto rapporto esistente tra i repubblicani nord-irlandesi e l'organizzazione

Eoin, Sinn Féin and the Politics of Left Republicanism, London, Pluto Press, 2009; LAGONEGRO, Giovanni, Storia politica di Euskadi Ta Askatasuna e dei Paesi Baschi, Milano, Tranchida, 2005; BOTTI, Alfonso, La questione basca: Dalle origini allo scioglimento di Batasuna, Milano, Mondadori, 2003; MELLUCCI, Alberto, DIANI, Mario, op. cit.

${ }^{21}$ PETRICOLA, Elena, I diritti degli esclusi nelle lotte degli anni settanta: Lotta Continua, Roma, Edizioni Associate, 2002, pp. 100-101.

22 «Irlanda. Lotta di popolo armata!», in Lotta Continua, 1/1972, pp. 14-17.

23 Situata al confine meridionale con la Repubblica d'Irlanda, è una delle sei contee che costituiscono l'Ulster britannico.

24 «Irlanda: offensiva dell'IRA», in Lotta Continua, 16 Aprile 1972.

25 FISK, Robert, «International Terrorist Activity in Europe», in The Times, 21 September 1972.

26 INSTITUTE FOR THE STUDY OF TERRORISM, IRA, INLA: Foreign Support and International Connections, London, Institute for the Study of Terrorism, 1988. 
italiana ${ }^{27}$. A fugare, poi, i dubbi rimasti provvederà l'intervista che uno dei militanti dell'IRA in sciopero della fame, detenuto presso il carcere di massima sicurezza di Maze, concesse nel novembre del 1972 all'inviata del giornale a Belfast; prima corrispondente straniera ad incontrare personalmente i detenuti in sciopero ${ }^{28}$. Sebbene il rapporto con l'Irlanda fosse privilegiato, una certa attenzione fu dedicata anche ad altre questioni nazionali irrisolte, e in certi casi si diede notizia, per la prima volta nel nostro paese, di programmi e riferimenti teorici di alcuni di questi nuovi soggetti politici. In virtù di questo interesse, comparvero diversi articoli, di alto valore storico e giornalistico, dedicati al Front de Libération du Québec, all'Armée Républicaine Bretonne e allo Scottish National Party ${ }^{29}$.

Anche Potere Operaio (PotOp) negli stessi anni si interesserà alle organizzazioni nazionalitarie attive nell'occidente europeo. Nel 1968 si tenne a Roma un primo incontro esplorativo tra membri, anche in questo caso, del movimento repubblicano nord-irlandese e PotOp33; fu però con il Convegno Internazionale di Firenze, nell'ottobre 1971, che questi rapporti vennero ufficializzati. Alle giornate fiorentine, organizzate presso l'Istituto Stensen, presero parte rappresentanti di diversi gruppi della sinistra radicale internazionale, tra cui alcuni membri dell'IRA ${ }^{31}$. Proprio la partecipazione dei militanti irlandesi destò un certo scandalo sulla stampa britannica, attirando diverse critiche. Il «Daily Express», qualche mese più tardi, attaccò violentemente PotOp per aver permesso ad un membro dell'IRA di parlare per tre ore senza nessun intervento da parte delle forze dell'ordine italiane ${ }^{32}$.

27 «Irlanda: la lotta armata», in Lotta Continua, 28 Aprile 1972. Durante l'estate una delegazione di Lotta Continua si recherà in Ulster per partecipare ad una serie di incontri pubblici organizzati dal movimento nazionalista irlandese. Resoconti quotidiani sull'attività della delegazione furono pubblicati per tutto il mese di Luglio del 1972.

28 «Nella Auschwitz irlandese 29 militanti della libertà stanno morendo di fame», in Lotta Continua, 2 Novembre 1972. Si veda inoltre «Lettera dall'Irlanda», in Lotta Continua, 22 Ottobre, 1972.

29 «Come lo zio Sam ingrassa sul sangue del Quebec. Storia di un saccheggio coloniale. Parla l'FLQ: Liberazione attraverso la lotta armata», in Lotta Continua, 17 Maggio 1972; «Bretagna: lotta unita di contadini e operai. Mobilitati i proletari della colonia interna», in Lotta Continua, 10 Giugno 1972; «Scozia. Dio salvi la regina!», in Lotta Continua, 15 Ottobre 1972.

$3^{\circ}$ GRANDI, Aldo, La generazione degli anni perduti: Storie di Potere Operaio, Torino, Einaudi, 2003, p. 267.

${ }^{31}$ Corte d'Assise di Roma, Verso la conferenza d'organizzazione per una nuova internazionale rivoluzionaria - Materiali del convegno internazionale organizzato da Potere Operaio, Firenze, 1-2-3 ottobre 1971, Procedimento n. 1067/79 contro Antonio Negri et altri, vol. XXVII, cartella 63 , fascicolo 5 , p. 2.

${ }^{2}$ Il quotidiano inglese dava rilievo alla notizia secondo cui, alcuni mesi prima, su chiamata di Potere Operaio, esponenti rivoluzionari di otto paesi si erano riuniti a Firenze per coordinare e intensificare le attività terroristiche in Europa e in America Latina. Il giornale sottolineava che tra gli interventi ce n'era stato uno in particolare, quello di un generale dell'Ira, durato oltre tre ore. Cfr. GRANDI, Aldo, op. cit., p. 268. 
Non tutti i membri del gruppo erano entusiasti di queste relazioni. Molti disprezzavano profondamente il "terzomondismo", convinti che l'operaismo più radicale fosse la sola valida chiave interpretativa dei conflitti:

\begin{abstract}
$\mathrm{Al}$ convegno internazionale di Firenze - racconta Giovanni Contini - organizzato da Lapo Berti, intervennero formazioni che parlavano linguaggi diversi. Era gente che, tra l'altro, certe cose le faceva mentre noi le dicevamo. [...] Sentii che il convegno non rappresentava urgenze nostre; erano, le loro, esperienze parallele, anche perché noi eravamo fortemente operaisti mentre queste formazioni erano tutto fuorché tali. Noi avevamo un grande disprezzo per tutto quello che era terzomondismo. Non credevamo che il Terzo Mondo potesse fare la rivoluzione. Pensavamo che tutto si sarebbe risolto nel centro del sistema capitalista e non nella periferia33.
\end{abstract}

Altri, invece, vedevano di buon occhio questi movimenti, dedicando attenzioni crescenti a fenomeni di questo tipo. Lapo Berti, e soprattutto Franco Piperno, dedicarono ampio spazio su «Potere Operaio del Lunedì» alla questione irlandese e alle lotte nazionalitarie in genere. Lo stesso Piperno si recherà di persona a Belfast nell'autunno del 1971 per studiare l'organizzazione dell'IRA e il lavoro svolto dai militanti repubblicani all'interno del movimento di occupazione delle case ${ }^{34}$. Sebbene piuttosto critico, l'interesse verso i nazionalismi periferici dei dirigenti di PotOp crebbe. Inizialmente le obiezioni furono molte; più di una volta si definirono superate e anacronistiche tanto le analisi quanto le prospettive della sinistra rivoluzionaria irlandese, accusata di essere portatrice di un coacervo di istanze nazionaliste di stampo conservatore35. Ad una parziale revisione delle critiche contribuì quasi certamente la serie di incontri, tenutisi in Ulster nel corso del 1972, che portarono Antonio Ceccotti ad incontrare, tra gli altri, Gerry Adams, leader in ascesa del movimento Provisional, che proprio in quegli anni si stava avvicinando al marxismo ${ }^{36}$. Diversi approfondimenti legati al tema nazionalitario comparvero in quel periodo sul periodico del gruppo, molti

\footnotetext{
33 Ibidem.

34 GRANDI, Aldo, op. cit., p. 267.

35 «Il guaio è che le differenze teoriche e politiche tra la sinistra marxista dell'Europa continentale e la sinistra, marxista e non, delle due Irlande è talmente grande da renderne in un primo momento molto difficile la comprensione. In realtà anche sul loro terreno più avanzato le sinistre irlandesi sembrano basarsi su analisi e prospettive politiche già da tempo superate e anacronistiche. Unità nazionale e indipendenza nazionale: questi sono il massimo orizzonte politico che i socialdemocratici Provisionals e i socialisti Officials sono riusciti e riescono e concepire». Cfr. «Il mezzogiorno del nord», in Potere Operaio del Lunedi, 3 Dicembre 1972. ${ }^{36}$ Sulla politica perseguita da Gerry Adams si rimanda a ENGLISH, Richard, op. cit.; ID., Terrorism: How to Respond, Oxford, Oxford University Press, 2009; MOLONEY, Ed, La storia segreta dell'IRA, Milano, Baldini Castoldi, 2004.
} 
dei quali riconducibili alla firma di Franco Piperno. Indubbiamente brillante fu l'analisi del caso gallese; si delineò, difatti, in modo molto preciso la rottura, avvenuta all'inizio degli anni '70, tra gli interessi della classe operaia e il Partito Laburista37, che comporterà una vera e propria ri-esplosione nazionalista in Galles, con un conseguente spostamento a sinistra del baricentro politico del Plaid Cymru ${ }^{38}$. Gli interrogativi che PotOp si pose, nel rapportarsi a questi movimenti, furono sostanzialmente due: «È possibile, oggi, un'utilizzazione rivoluzionaria del nazionalismo? Ed è possibile qui in Europa tale utilizzazione39?». Se la massima solidarietà veniva espressa per il riconoscimento delle lingue minoritarie, diverse remore persistevano sul piano delle rivendicazioni istituzionali e politiche, che partiti e movimenti nazionalitari avanzavano spesso senza una particolare coerenza. A prevalere, però, fu la ferma volontà di cercare un confronto con quanti iniziavano ad essere considerati dei nuovi possibili soggetti rivoluzionari40. Nel 1973 un fitto scambio di corrispondenza tra l'ufficio internazionale di Potere Operaio e i vertici del movimento indipendentista occitano Lutte Occitane, evidenziò la volontà del gruppo italiano di comprendere la natura politica di queste realtà. Emerse, con forza, la necessità di appurare se le rivendicazioni avanzate da questo genere di partiti fossero di una tipologia meramente nazionalista, o se, invece, potessero rientrare nel novero delle forze fautrici di una effettiva rottura di classe ${ }^{41}$. Nonostante gli sforzi della commissione internazionale, però, la ferma opposizione

37 «[...] Finché è durato lo sviluppo economico del Galles il nazionalismo, sempre latente, è rimasto assorbito dall'ideologia laburista. Ma quando le miniere hanno cominciato a dare segni di esaurimento e quando sono entrate in crisi molte fabbriche locali schiacciate dalla concorrenza dei grandi poli industriali dell'Inghilterra centro-meridionale, ha cominciato a delinearsi una frattura fra gli interessi materiali dei proletari gallesi e il partito laburista che andava man mano perdendo i suoi connotati riformistici e diventava la punta di diamante del programma di ristrutturazione (leggi di disoccupazione e emigrazione) dell'economia gallese». Cfr. «Galles: dal nazionalismo alla lotta di classe», in Potere Operaio del Lunedì, 17 Dicembre 1972.

${ }^{8}$ Sulla storia politica del Plaid Cymru (letteralmente Partito del Galles) si rimanda a LYNCH, Peter, «From Red to Green: The political Strategy of Plaid Cymru in the 1980s and 1990s», in Regional \& Federal Studies, vol. 5, 1995, pp. 197-210; ID., Minority Nationalism and European Integration, Cardiff, University of Wales Press, 1996; DE WINTER, Lieven, TÜRSAN, Huri, Regionalist Parties in Western Europe, London-New York, Routledge, 1998; GABEL, Matthew J., HUBERT, John, «Putting Parties in Their Place: Inferring Party Left-Right Ideological Positions From Party Manifestos Data», in American Journal of Political Science, vol. 44, 2000, pp. 94-103; MCALLISTER, Laura, Plaid Cymru: The Emergence of a Political Party, Bridgend, Seren. Meguid, 2001.

39 «Questione nazionale e salario garantito», in Potere Operaio del Lunedì, 28 Gennaio 1973.

40 «Queste lotte - nella misura in cui attaccano lo Stato del capitale e incidono direttamente sulla sua funzione di organizzatore centrale delle operazioni di dislocazione e mobilizzazione della forza lavoro (e dunque di scomposizione dell'unità politica di classe operaia) - ci riguardano molto da vicino». Cfr. «La questione nazionale in Europa: Alla crescente egemonia degli strati proletari nelle lotte nazionali corrisponde l'uso sempre più largo di forme di lotta armata» in Potere Operaio del Lunedì, 28 Gennaio 1973.

41 «Occitania: Volem vivre al pais», in Potere Operaio del Lunedi, 4 Febbraio 1973. 
della componente maggioritaria, e decisamente anti-terzomondista, del gruppo non venne scalfita e le relazioni che in quei primi anni erano state intessute, non si svilupparono ulteriormente. Anche alla luce di quanto appena detto si può spiegare l'assoluta mancanza di interesse da parte di Potere Operaio, così come di Lotta Continua, nei confronti dei movimenti di ispirazione nazionalitaria presenti all'interno dei confini italiani. Nemmeno una parola, infatti, fu spesa sulla contemporanea riorganizzazione, seppure effimera, di movimenti di questo genere in Friuli e in Sardegna.

\section{I diritti delle nazioni proibite. La politica nazionalitaria di Democrazia Proletaria}

Ad invertire la rotta in maniera dirompente provvederà, qualche anno dopo, il neonato progetto di Democrazia Proletaria (DP). Nel tentativo di tirare le fila di ciò che rimaneva della sinistra rivoluzionaria, nel 1978, durante i lavori dell'assemblea costituente del nuovo soggetto demoproletario, si mise in discussione l'idea stessa di partito strutturato "nazionalmente". I delegati delle federazioni friulana, sarda, valdostana, altoatesina e delle comunità albanesi della Calabria, presentarono una mozione che invitava il partito ad assumere i problemi e le aspirazioni delle identità nazionali, nazionalitarie ed etnico-lingistiche delle minoranze esistenti nello stato italiano ${ }^{42}$. La componente sarda fu la prima a rompere gli indugi, denunciando l'inefficacia del sistema autonomistico fin lì sperimentato sull'isola43:

In questa fase pertanto dobbiamo essere capaci di aprire una campagna politica per l'allargamento degli spazi dell'autonomia regionale, anche se il nostro obiettivo strategico rimane un regime di autonomia, più precisamente di reale autogoverno economico e sociale, ben diverso e più avanzato di quello attuale, all’interno di un

\footnotetext{
42 A riguardo si veda GAMBETTA, William, Democrazia Proletaria: La nuova sinistra tra piazze e palazzi, Milano, Punto Rosso, 2010, p. 138.

43 In quegli anni in Sardegna si registrò una notevole crescita dell'attività di gruppi autonomisti e indipendentisti. Proprio tra la fine del 1977 e l'inizio del 1978 si palesò il primo gruppo terroristico composto esclusivamente da sardi: Barbagia Rossa. Furono, però, diverse le formazioni nazionalitarie che godettero, all'inizio degli anni '8o, di un certo sostegno e di una qualche notorietà. Una serie di buoni risultati elettorali conseguiti dal Partito Sardo d'Azione, insieme alla nascita di gruppi come Su Populu Sardo e il Partidu Sardu Indipendentista Sotzialista Libertariu, spinsero alcuni a parlare di "nuovo vento sardista". Cfr. SOTGIU, Girolamo, op. cit.; CONTU, Gianfranco, La questione nazionale sarda, tra autonomismo e indipendentismo, Quartu, Alfa, 1990.
} 
processo che rompa con i meccanismi della politica economica e amministrativa, accentratrice e uniformista, dello Stato italiano falsamente regionalista. 44

La volontà di saldare la tradizione sardista di sinistra, di cui espressione fu Emilio Lussu, con quella comunista rivoluzionaria di Gramsci, costituì il progetto politico di quei militanti sardi che videro nel nuovo partito un perfetto interlocutore per il rilancio di un programma, ambizioso e contraddittorio allo stesso tempo, di stampo socialistanazionalitario. Le altre federazioni coinvolte, espressione di altrettante comunità minoritarie, si inserirono in questo dibattito evidenziando il processo di espropriazione economica, culturale e linguistica messo in atto dallo stato italiano nei confronti delle minoranze etnico-nazionali, e denunciando, senza giri di parole, la politica accentratrice dello stato 45 . Non mancarono, ovviamente, una serie di critiche all'atteggiamento tenuto fino ad allora dalla sinistra ufficiale:

In questo contesto, compito della Costituente di DP è quello di superare i gravi ritardi del dibattito su queste tematiche della sinistra italiana e di inserirle fra quelle di cui il movimento operaio deve farsi carico, all'interno di un progetto complessivo che tenda alla trasformazione socialista e rivoluzionaria della società. 46

Dalla volontà di colmare questo ritardo iniziò il percorso che condurrà al convegno sul tema "Nazionalità, minoranze, lotta di classe in Europa oggi”, tenutosi a Cagliari nel febbraio del 1979. Ad emergere con forza dal convegno sardo fu una nuova lettura delle componenti sociali del paese. Le minoranze nazionali e linguistiche vennero considerate portatrici di una forte vocazione ribellistica repressa e quindi gli si attribuì lo status di nuovi soggetti rivoluzionari. Mario Brunetti, esponente calabrese del partito, nel corso di una delle relazioni presentate in quei giorni, chiarirà molto bene questo nuovo approccio:

Sono in molti ad esprimere meraviglia per questo risveglio forte delle rivendicazioni minoritarie, ma esso è frutto di un vuoto di interpretazioni dei fermenti che si agitano nella società e dei nuovi movimenti che caratterizzano lo

\footnotetext{
44 Archivio Storico Marco Pezzi, Fondo Democrazia Proletaria, fasc. Dp nazionale. Congressi, sottofasc. Documenti I Congresso, Mozione sulla "Questione Sarda" presentata dalla federazione di Democrazia Proletaria della Sardegna e allegata agli atti dell'assemblea costituente di Dp e Mozione al congresso dei compagni delle federazioni friulana, sarda, valdostana, altoatesina e dei compagni delle comunità albanesi della Calabria (approvata come parte integrante della mozione finale), documento dattiloscritto, s.d., [1978].

45 Ibidem.

46 Ibidem.
} 
scenario europeo e italiano. [...] La contemporaneità tra quello che è stato definito il risveglio del conflitto etnico-linguistico e la nascita dei nuovi movimenti non è accidentale, poiché essi sono entrambi espressione di un medesimo fenomeno di crisi ed entrambi esprimono l'esigenza della costruzione di una società diversa, meno fagocitante ed oppressiva. 47

Il convegno attirò diverse critiche - si arrivò a denunciare il pericolo corso dall'unità dello Stato - che si intensificarono dopo che nell'aprile del 1979 si tenne l'ultimo di una serie di incontri organizzati dalla Commissione per le Questioni Internazionali, diretta da Luigino Scricciolo48. Agli incontri parteciparono gruppi provenienti da diverse nazioni europee, ma fu la presenza del Euskal Iraultzako Alderdia (EIA, Partito per la Rivoluzione Basca, braccio politico di ETA poli-mils), del Sinn Féin irlandese (strettamente connesso all'IRA Provisional) e del Unvaniezh Demokratel Breizh (UDB, Unione Democratica Bretone, partito vicino all'ARB), a destare un certo scandalo; attirando, nuovamente, l'attenzione delle forze dell'ordine e della magistratura.

La componente nazionalitaria del partito, sempre più consistente, raccolse, poi, un importante successo nel corso del III Congresso nazionale, tenutosi a Milano nel luglio del 1982. Alle federazioni che si erano fatte portatrici di istanze autonomiste, infatti, venne riconosciuta piena libertà decisionale:

\begin{abstract}
All'interno del complesso progetto di trasformazione socialista della società, D.P. riconosce la potestà che si organizzi in forma autonoma la presenza politica nei territori dello stato italiano caratterizzati dall'esistenza di minoranze nazionali, di nazionalità oppresse o non riconosciute, o di situazioni consolidate di autonomia. A tali forme organizzative autonome spetta la nomina di una propria rappresentanza nell'organismo dirigente unitario che quindi assume una caratteristica di plurinazionalità. 49
\end{abstract}

Il partito assunse, così, per la prima volta nella storia politica italiana, una struttura federale, ufficializzando l'autonomia di DP sarda, DP friulana (la cui denominazione mutò in Partit Furlan pal Socialism), DP trentina, DP Sudtirolo (da quel momento

47 CAVALLO, Giorgio, «Minoranze, nazionalità, lotta di classe in Europa oggi», in Unità Proletaria, a. IV, 1, Febbraio-Marzo 1979, pp. 52-57.

48 GAMBETTA, William, op. cit., p. 176.

49 Archivio Storico Marco Pezzi, Fondo Democrazia Proletaria, fasc. Dp nazionale. Congressi, sottofasc. Documenti III Congresso, Atti Congressuali - Terzo Congresso Nazionale Democrazia Proletaria Milano 1-4 Luglio 1982, documento dattiloscritto, 1982. 
Arbeiter Demokratie) e Nuova Sinistra Valdostana50. L'autonomia di queste federazioni non fu mai messa seriamente in discussione, anzi venne ribadita nel corso dei congressi successivi.

In seguito, l'analisi della situazione continentale, alla luce dei processi d'integrazione europea, convinse il partito della necessità di ribadire la ferma opposizione tanto al modello occidentale quanto a quello sovietico. Si decise di optare per un progetto alla base del quale porre l'idea di un'Europa «socialista autogestionaria dei lavoratori, delle nazionalità e delle regioni - comprese le nazionalità oggi non riconosciute dagli stati (sardi, baschi, corsi, ecc.)»51. Un modello così concepito comportò l'ulteriore avvicinamento di un partito come DP alle coordinate politiche promosse negli stessi anni dai movimenti di liberazione nazionale, impegnati a promuovere la costruzione di un'Europa federale. Un'unione, però, di stampo socialista, che ponesse al centro della discussione i bisogni dei lavoratori in una chiave fermamente anticapitalista. In quest'ottica si colloca l'approvazione delle statuto definitivo di Democrazia Proletaria, nel corso del VI Congresso nazionale, con il quale si inserì ufficialmente la realizzazione di uno stato socialista di carattere federale, tra gli obiettivi del partito. Nel preambolo dello statuto adottato a Riva del Garda nel maggio del 1988 si legge:

Dp si batte per il diritto all'autodeterminazione dei popoli e la piena sovranità delle nazionalità non italiane, riconosciute e non riconosciute, presenti nell'ambito degli attuali confini dello Stato. Dp afferma altresì l'opzione federalista come orizzonte che regola il rapporto tra nazionalità e minoranze linguistiche ed etniche, autonomie locali nella prospettiva del superamento delle barriere statali e di tutti gli ostacoli che si frappongono al pieno sviluppo delle relazioni fra i popoli.52

Alla voce "I partiti federati":

${ }^{50}$ I risultati elettorali nelle regioni appena menzionate premiarono la scelta di DP, che raccolse un buon numero di voti: il 2,3\% in Trentino Alto Adige e il 2,0\% in Friuli Venezia Giulia. Un dato confortante, all'interno di quella che resta una disfatta elettorale, arrivò anche da alcuni centri importanti: Trento (3,6\%), Bolzano (3\%) e Oristano (2,9\%). Cfr. GAMBETTA, William, op. cit., pp. 82-83.

${ }^{1}$ Archivio Storico Marco Pezzi, Fondo Democrazia Proletaria, fasc. Dp nazionale. Congressi, sottofasc. Documenti V Congresso, Al bivio del Duemila: Idee e progetti per l'alternativa. Tesi approvata dal $5^{\circ}$ Congresso Nazionale Democrazia Proletaria - Palermo 22-27 Aprile 1986.

${ }^{2}$ Archivio Storico Marco Pezzi, Fondo Democrazia Proletaria, fasc. Dp nazionale. Congressi, sottofasc. Documenti VI Congresso, Lo statuto di Dp, 1988. 
Art 6.1 I rapporti tra Dp della nazionalità italiana maggioritaria nello Stato e i partiti organizzati nei territori dello Stato ove vivono minoranze nazionali, nazionalità oppresse e non riconosciute, oppure vi sia una consolidata tradizione di autonomia, sono regolati da un patto federativo che si ispiri ai principi generali del presente statuto. 53

Il percorso storico-politico di DP, però, stava avviandosi inesorabilmente alla conclusione, e il 1989 ne sancì la crisi definitiva. Il crollo del blocco sovietico tolse di scena un indiscusso protagonista della storia novecentesca, mandando in crisi il sistema di riferimento ideologico di molti partiti occidentali. Al contempo numerosi conflitti di tipo nazionalista esplosero, o ri-esplosero, sullo scenario politico europeo. L'Europa e l'occidente, si trovarono ad assistere quasi con imbarazzo ad un'improvvisa quanto inaspettata riproposizione della "questione nazionale", che rischiava, tra l'altro, di ostacolare il processo di riassestamento globale. Il timore che un revival nazionalista fosse portatore di istanze di tipo xenofobo ed esclusivista - nella ex-Yugoslavia come in tutto il blocco orientale - costrinsero la nuova sinistra italiana, giunta ormai al termine del proprio percorso, ad una revisione, almeno parziale, della propria posizione su queste tematiche. Analisi, questa, condotta nel corso del Seminario Nazionale Esteri, tenutosi a Roma l'11 e 12 novembre del $1989^{54}$. Il timore di una inarrestabile diffusione di sentimenti razzisti all'interno del nuovo assetto continentale, spingerà i demoproletari a preannunciare il rilancio di una politica effettivamente internazionalista e solidaristica, mirante all'arginamento dell'intransigenza nazionalista, apparentemente in ascesa. I rischi insiti nell'esasperazione delle diversità etnico-nazionali, alimentati dal succedersi degli eventi nella ex-Jugoslavia, uniti allo spostamento a destra dei movimenti nazional-populisti emersi dalle macerie sovietiche, posero fine alla politica nazionalitaria del partito. Il successivo auto-scioglimento di DP, decreterà la definitiva scomparsa dal panorama politico italiano, dell'unico partito che si sia mai strutturato in maniera federativa, relegando ancora una volta nel dimenticatoio il dibattito sui nazionalismi periferici nel nostro paese.

\footnotetext{
53 Ibidem.

54 «La crisi dei regimi dell'est, si legge nella sintesi dei lavori, ci consegna anche un'altra questione sulla quale è urgente un approfondimento: quella delle nazionalità, e della ricostruzione, nella crisi delle idealità transnazionali e internazionaliste, di nuove forti identità nazionali o comunitarie non solo come risposta ad un'oppressione nazionale (Paesi Baschi, Irlanda, regioni baltiche dell'URSS, Kossovo) ma come etnocentrismo esasperato e potenzialmente oppressore (Azerbaijan, panserbismo, rischio di pangermanesimo)». Cfr. Archivio Storico Marco Pezzi, Fondo Democrazia Proletaria, fasc. Dp nazionale. Congressi, sottofasc. Documenti VII Congresso, Sintesi del seminario Nazionale Esteri - Roma 11-12 Novembre 1989, documento dattiloscritto, 1989.
} 
Dalla scomparsa di Democrazia Proletaria si è innescato un processo di appropriazione, in taluni casi improprio, di temi come federalismo, autonomismo e autodeterminazione, da parte di alcuni partiti di nuova formazione; si consideri, a tal proposito, il caso della Lega Nord. Le stesse parole d'ordine care alla sinistra rivoluzionaria, una volta innestate su di un programma di stampo esclusivista, hanno visto modificato, quando non stravolto, il proprio intrinseco significato. Lungi da chi scirve sottovalutare l'importanza della nascita di "nuove" forme partitiche sul continente, come il fenomeno leghista in Italia o il Vlaams Blok in Belgio, ma allo stesso tempo la notevole ascesa di movimenti nazionalitari di sinistra, come il Sinn Fèin, Herri Batasuna o Esquerra Republicana de Catalunya, ha lasciato di stucco tanto gli osservatori quanto gli studiosi. Ricostruire la storia di questi anomali movimenti politici ha riportato all'interno del dibattito storiografico lo studio delle commistioni tra le rivendicazioni prettamente autonomistiche e i temi cari alla sinistra internazionale, come l'ecologismo, il terzomondismo e la giustizia sociale. Alla luce di ciò acquista ancora più valore il monito lanciato da Benedict Anderson; e agli storici non resta che raccogliere la sfida e cercare di colmare quel vuoto per troppo tempo volutamente ignorato. 


\section{* L'autore}

Laureatosi in storia presso l'Università della Calabria con una tesi sui rapporti tra socialismo e nazionalismo nell'Irlanda di inizio Novecento, è attualmente dottorando in Scienze storiche in età contemporanea presso l'Università degli Studi di Siena.

Studia i nazionalismi periferici dell'Europa Occidentale, avendo, inoltre, svolto ricerche storiografiche presso il Trinity College of Dublin, l'International Institute of Social History of Amsterdam, il National Archives of Dublin, l'International Conflit Research Institute of Belfast e la Universidad del Pais Vasco-Euskal Herriko Unibertsitatea di Bilbao.

Responsabile dell'Osservatorio sulle Politiche Comunitarie in tema di Minoranze (OsPCoM) dell’Università degli Studi di Siena.

Ha pubblicato un saggio dal titolo: «Un punto di svolta nella questione irlandese: il socialismo gaelico e il pensiero di James Connolly», in Storia e Futuro, 27, 2011, http://www.storiaefuturo.com/it/numero_27/articoli/1_irlanda-socialismo-gaelicoconnolly 1438.html.

URL: < http://studistorici.com/progett/autori/\#Perri >

\section{Per citare questo articolo:}

PERRI, Paolo, «Nazionalità e lotta di classe. La Nuova Sinistra e i nazionalismi periferici», Diacronie. Studi di Storia Contemporanea: Quando la classe operaia andava in paradiso, 13/2/2012,

URL:< http://www.studistorici.com/2012/02/13/perri_numero_9/ >

Diacronie Studi di Storia Contemporanea $\widehat{\beta}$ www.diacronie.it

Risorsa digitale indipendente a carattere storiografico. Uscita trimestrale. redazione.diacronie@hotmail.it

Comitato di redazione: Marco Abram - Giampaolo Amodei - Jacopo Bassi - Luca Bufarale - Alessandro Cattunar - Alice De Rensis Barbara Galimberti - Deborah Paci - Fausto Pietrancosta - Martina Sanna - Matteo Tomasoni - Luca Zuccolo 